\title{
Parasites in Duodenal Aspirate using Endoscopy in Sohag, Egypt
}

\section{Hanaa A. El-Hady ${ }^{1}$, Samir A. Abd-Elmaged ${ }^{2}$, Amal A. Abd- Elmawgood $^{1}$, Salwa G. Ahmed ${ }^{1}$.}

Departments of Medical Parasitology ${ }^{1}$, General Surgery ${ }^{2}$, Faculty of Medicine, Sohag University, Egypt.

Correspondence to Salwa G. Ahmed, Department of Medical Parasitology, Faculty of Medicine, Sohag University, Egypt

E-mail: salwa.gamal@yahoo.com.

\begin{abstract}
:
Background and study aim: Gastrointestinal parasites cause infectious diseases. Parasitic infection of duodenum is a relevant cause of its inflammation and appearance of upper GIT manifestation, especially in developing countries. Examination of duodenal aspirates can be very helpful in diagnosing intestinal infection because it eliminates some of the problems inherent in stool examination. The aim of this study was to estimate the prevalence of parasites in duodenal aspirate obtained by upper gastrointestinal tract endoscopy.

Subjects and methods: This cross-sectional study was conducted from March 2017 to March 2018 in sohag university hospital and Center of the digestive system. It was done on 100 patients who underwent Esophago-gastro-duodenoscopy. A total of 100 duodenal aspirate samples were collected from patients were subjected to Esophagogastroduodenoscopy for diagnostic or therapeutic reason. All cases were reviewed as regard age, sex. Cases with parasitic infestations were characterized regarding the mentioned variables.

Results: $60 \%$ of duodenal aspirate samples were positive. Monoparasitized patients constituted $38 \%$ (38/100) of patients while $22 \%$ were polyparasitized. There were 44 patients (44\%) of Cryptosporidium infection, 21 patients (21\%) of Blastocystis hominis infection, 11 patients (11\%) of Cyclospora infection, 8 patients (8\%) of Giardia lamblia infection.
\end{abstract}

Conclusion: We concluded that the duodenal aspirate samples obtained during gastrointestinal endoscopic procedures helped in diagnosing the parasites.

Keywords: intestinal parasites, duodenal aspirate, Esophagogastroduodenoscopy.

\section{Introduction:}

Gastrointestinal parasites cause infectious diseases, their prevalence is highest in areas of intense poverty in low- and middle-income countries in the tropical and subtropical regions of sub-Saharan Africa, Asia, and Latin America and the Caribbean [1-4]. Parasitic infection of duodenum is a relevant cause of its inflammation and appearance of upper GIT manifestation. Moreover, parasitic diseases have acquired highlights in the context of immunosuppressive diseases, especially after the emergence of HIV pandemic and propagation of immunomodulation therapies. In such cases, intestinal

parasitosis can promote severe disease and lead to death [5].

Upper endoscopy is a very important tool for the diagnosis of gastrointestinal problems, and there are some reports of parasitic diagnosis during a routine upper endoscopy [6, 7]. Obtaining of duodenal fluid during the upper gastrointestinal endoscopy and microscopic examination of it can help in the diagnosis of parasites like Giardia lamblia especially in patients with persistent diarrhea [8]. also intestinal aspirates from immunocompromised patients show protozoa: Giardia, Isospora, 
MEDICAL JOURNAL

Vol. 22 No.3 October 2018
Parasites in Duodenal Aspirate using Endoscopy Salwa G. Ahmed
Cryptosporidia, and helminths Strongyloides stercoralis [9].

Examination of duodenal aspirates can be very helpful in diagnosing intestinal infection because it eliminates some of the problems inherent in stool examination, including less debris, ease of high-speed centrifugation, and easier recognition of parasite [10]. Staining of aspirate samples by methylene blue, iodine and Kinyoun's modified acid-fast is helpful in diagnosis, Also Antigen detection using Enzyme Linked Immuno-Assay can be done [11].

\section{Subjects and methods:}

This cross-sectional study was conducted from March 2017 to March 2018 in sohag university hospital and Center of the digestive system. It was done on 100 patients who underwent Esophago-gastro-duodenoscopy.

\section{Collection and preservation of} samples:

- 100 duodenal aspirate samples were collected from patients were subjected to Esophagogastroduodenoscopy for diagnostic or therapeutic reason.

- Samples were aspirated from the first and the second portion of the duodenum using EGD and were collected in a clean tight fitting container. Then every sample was labeled properly with the patient's name. Half of the amount of each sample was added to it sodium acetate-acetic acid-formalin solution.

- The endoscope used was disinfected after each introduction, with Cidex.

Microscopic examination of samples: After performing concentration by centrifugation of the fluid at $500 \times \mathrm{g}$ for $5 \mathrm{~min}$ and the supernatant was decanted. Microscopic examination was done on the sediment as wet mount and after staining the samples with Modified Kinyoun's acid-fast stain for detection of oocysts of coccidian.

\section{Statistical analysis:}

Results were gathered, organized and tabulated in an Excel 2013 spreadsheet. Data were analyzed using IBM SPSS Statistics for Windows version 25.0. Quantitative data were expressed as mean \pm standard deviation, median, and range. Qualitative data were expressed as number and percentage. The data were tested for normality using the Shapiro-Wilk test. The nonparametric Mann-Whitney test was used for data which wasn't normally distributed. Chi-square $(\chi 2)$ test and Fisher's Exact Test were used for comparison regarding qualitative variables as appropriate. A 5\% level was chosen as a level of significance in all statistical tests used in the study.

\section{Results:}

The demographic data: The age of patients ranged from 18 to76 years with a mean of $48.48 \pm 14.68$. Males constituted $53 \%$ (53 patients) of the whole patients, while females were $47 \%$ (47 patients) of them.

In the current study, 59\% of cases, the indications for EGD was the diagnostic reason, $41 \%$ of cases were done for the therapeutic reason for liver cirrhosis and esophageal varices for banding.

$60 \%$ of duodenal aspirate samples taken from 100 cases that examined for direct parasite examination were positive. Monoparasitized patients constituted 38\% (38/100) of patients while $22 \%$ were polyparasitized. Table (1) 
MEDICAL JOURNAL

Vol. 22 No.3 October 2018
Parasites in Duodenal Aspirate using Endoscopy Salwa G. Ahmed

Table (1): Rate of infection among the studied patients according to the results of the duodenal aspirate analysis $\left(N_{.}=100\right)$

\begin{tabular}{|c|c|}
\hline Infection & No. $(\%)$ \\
\hline \hline Positive $(60(60 \%))$ & \\
Mono parasitism & $\mathbf{3 8}(\mathbf{3 8 \%})$ \\
Multi parasitism & $\mathbf{2 2}(\mathbf{2 2 \%})$ \\
\hline \hline Negative & $\mathbf{4 0}(\mathbf{4 0 \%})$ \\
\hline
\end{tabular}

A total of 100 duodenal aspirate samples, there were 44 patients (44\%) of Cryptosporidium infection, 21 patients $(21 \%)$ of Blastocystis hominis infection, 11 patients (11\%) of Cyclospora infection, 8 patients $(8 \%)$ of Giardia lamblia infection. Table (2).

Table (2): Distribution of the studied patients according to detected parasites in duodenal aspirate $\left(\mathbf{N}_{.}=\mathbf{1 0 0}\right)$

\begin{tabular}{|l|l|}
\hline Parasites in duodenal aspirate & $\begin{array}{l}\text { Positive } \\
\text { No. }(\%)\end{array}$ \\
\hline \hline Cryptosporidium & $44(44 \%)$ \\
\hline \hline Blastocystis hominis & $21(21 \%)$ \\
\hline \hline Cyclospora & $11(11 \%)$ \\
\hline \hline Giardia lamblia & $8(8 \%)$ \\
\hline
\end{tabular}

The study show that Cryptosporidium, Cyclospora, Giardia lamblia, and Blastocystis hominis were in high rate in age group 40-60 years as we found that in this age group had about two third of Cryptosporidium cases, $50 \%$ of Giardia lamblia cases, $45 \%$ of cyclospora cases and about $52 \%$ of Blastocystis hominis cases from all result. Table (3) Table (3): The relation between the type of parasite in duodenal aspirate and age groups.

\begin{tabular}{|c|c|c|c|c|}
\hline \multirow[b]{2}{*}{ Age group } & \multicolumn{4}{|l|}{ Parasites } \\
\hline & $\begin{array}{l}\text { Cryptosporidium } \\
\text { No. } 44\end{array}$ & $\begin{array}{l}\text { Blastocystis hominis } \\
\text { No. } 21\end{array}$ & $\begin{array}{l}\text { Cyclospora } \\
\text { No. } 11\end{array}$ & $\begin{array}{l}\text { Giardia lamblia } \\
\text { No. } 8\end{array}$ \\
\hline$<20$ years & $0(0.0 \%)$ & $0(0.0 \%)$ & $1(9.1 \%)$ & $0(0.0 \%)$ \\
\hline \begin{tabular}{|l|}
$20-40$ \\
Years
\end{tabular} & $10(22.7 \%)$ & $4(19 \%)$ & $0(0.0 \%)$ & $3(37.5 \%)$ \\
\hline 40-60 years & $29(65.9 \%)$ & $11(52.38 \%)$ & $5(45.4 \%)$ & $4(50 \%)$ \\
\hline$>60$ Years & $5(11.3 \%)$ & $6(28.57 \%)$ & $5(45.4 \%)$ & $1(12.5 \%)$ \\
\hline
\end{tabular}

The relation between the type of parasite in duodenal aspirate and gender there is no significance, however, infection was higher in males than female in duodenal aspirate as Cryptosporidium (54.5\%), Cyclospora (72.7\%) and Giardia lamblia (62.5\%) while Blastocystis hominis equal between both male and female. Table (4)

Table (4): The relation between the type of parasite in duodenal aspirate and gender.

\begin{tabular}{|c|l|l|l|l||}
\hline Parasite & $\begin{array}{l}\text { Females } \\
\text { No. }(\%)\end{array}$ & $\begin{array}{l}\text { Males } \\
\text { No. }(\%)\end{array}$ & $\begin{array}{l}\text { Total } \\
\text { No. }(\%)\end{array}$ & P-value \\
\hline \hline Cryptosporidium & $\mathbf{2 0}(\mathbf{4 5 . 5 \% )})$ & $\mathbf{2 4}(\mathbf{5 4 . 5 \% )}$ & $\mathbf{4 4}(\mathbf{1 0 0 \%})$ & 0.784 \\
\hline \hline Blastocystis hominis & $\mathbf{1 1 ( 5 2 . 4 \% )}$ & $\mathbf{1 0 ( 4 7 . 6 \% )}$ & $\mathbf{2 1 ( 1 0 0 \% )}$ & 0.578 \\
\hline \hline Cyclospora & $\mathbf{3 ( 2 7 . 3 \% )}$ & $\mathbf{8 ( 7 2 . 7 \% )}$ & $\mathbf{1 1 ( 1 0 0 \% )}$ & 0.165 \\
\hline \hline Giardia lamblia & $\mathbf{3 ( 3 7 . 5 \% )}$ & $\mathbf{5 ( 6 2 . 5 \% )}$ & $\mathbf{8 ( 1 0 0 \% )}$ & $0.427 *$ \\
\hline \hline
\end{tabular}

P-value was calculated by the Chi-square test. * P - value was calculated by Fisher's Exact Test. Significant association $(\mathrm{P}-\mathrm{value}<0.05)$ 


\section{Discussion:}

Microscopic examination of duodenal aspirates or biopsy material may be indicated in patients with suspected cases as giardiasis, cryptosporidiosis, especially in whom stool examination is negative [12]. Because of the clear liquid nature of the duodenal aspirate considerably facilitates the recognition of trophozoites [13]. The present study has shown that examination of duodenal aspirate is a good method for the diagnosis of cryptosporidiosis, giardiasis, blastocystosis, and cyclosporiasis.

\section{Cryptosporidium infection}

In our study, Cryptosporidium cases were $44 \%$ in duodenal aspirate samples. This is compatible with result of a study done in Egypt found that Cryptosporidium prevalence among individuals with diarrhea visiting inpatient and outpatient clinics in Egypt ranges from 0\%-49\% [14]. Also In Al-Madinah, Saudi Arabia, cross-sectional study was done to study the prevalence of Cryptosporidium spp., in duodenal aspirate samples of 109 during routine upper endoscopy, Cryptosporidium were microscopy detected in $3 / 109(2.75 \%)$ and Cryptosporidium were molecularly detected in $7 / 109$ (6.4\%) in duodenal aspirate samples [11]. C. parvum is an obligatory intracellular intestinal parasite of human[15]. It could be a cause of upper GIT symptoms that should be considered during upper endoscopy and aspirate examination help in detection of that infection.

\section{Giardia lamblia infection}

In our study giardia cases were $8 \%$ in duodenal aspirate samples. This is compatible with a study was done in Egypt, on 120 patients with chronic dyspeptic symptoms, who attended the Internal Medicine outpatient clinic of the Kasr Al-Aini Faculty of Medicine. they were investigated by upper endoscopy and duodenal aspirates were collected from them to find that the prevalence of $G$. intestinalis among duodenal aspirates was 8 (6.66\%) [16]. another study was done in Tehran, Iran on a group of 130 dyspeptic patients who had undergone upper gastrointestinal endoscopy, had undergone duodenal aspiration and samples were positive for giardia among 2 (1.5\%) patients [17]. Increase number of sample may be the cause of this little difference in the percentage, however microscopic examination of duodenal content help in diagnosing cases of giardiasis [18].

\section{Blastocystis sp. infection}

It is one of the most common parasites found in the intestinal tract of man was considered to be a member of normal intestinal flora in the past, but in recent years it has been accepted as a very controversial pathogenic protozoan [19]. In our study, $21 \%$ of patients were found to be infected with Blastocystis hominis. although its habitat is large intestine but it could be found in duodenum as in a study that found 5 out of 15 patients infected with $B$. hominis were diagnosed through the recovery of the Blastocystis cyst from duodenal aspirate, That might have been related to the $\mathrm{pH}$ of the duodenal aspirate of those particular patients (4.0 \pm 2.34 ) because at such a $\mathrm{pH}$ value [13]. as protease activity of $B$. hominis showed $\mathrm{pH}$ dependence and it was highest at $\mathrm{pH} 7$ [20].

\section{Cyclospora infection}

It infects the gastrointestinal tract and causes acute diarrheal disease in human [21], especially affect children and immunocompromised patients [22]. In our study, Cyclospora cases were $11 \%$ in duodenal aspirate samples. Its diagnosis by duodenal aspirate examination is possible as in a study performed upper GIT endoscopy on 9 patients in Nepal identified as 
having Cyclospora diarrhea. The organism was found in 2 of 9 of duodenal aspirate. [23]

\section{Conclusion:}

We concluded that the duodenal aspirate samples obtained during gastrointestinal endoscopic procedures helped in diagnosing the parasites as it had clear nature that facilitates recognition of the named parasites.

\section{References:}

1. Hotez, P. J. (2008). The giant anteater in the room: Brazil's neglected tropical diseases problem. PLoS Negl Trop Dis, 2(1), e177.

2. Hotez, P. J. (2007). Neglected diseases and poverty in "The Other America": the greatest health disparity in the United States? PLoS Negl Trop Dis, 1(3), e149.

3. Hotez, P. J., \& Kamath, A. (2009). Neglected tropical diseases in subSaharan Africa: review of their prevalence, distribution, and disease burden. PLoS Negl Trop Dis, 3(8), e412.

4. Brooker, S., Clements, A. C., \& Bundy, D. A. (2006). Global epidemiology, ecology and control of soil-transmitted helminth infections. Advances in parasitology, 62, 221-261.

5. Santos, R. B. d., Fonseca Jr, L. E., Santana, A. T. d. A., Silva, C. A. C., \& Guedes, J. C. (2011). Clinical, endoscopic and histopathological profiles of parasitic duodenitis cases diagnosed by upper digestive endoscopy. Arquivos de gastroenterologia, 48, 225-230.

6. el Sheikh Mohamed, A. R., al Karawi, M. A., \& Yasawy, M. I. (1991). Modern techniques in the diagnosis and treatment of gastrointestinal and biliary tree parasites. Hepato-gastroenterology, 38(2), 180-188.

7. Kato, T., Kamoi, R., Iida, M., \& Kihara, T. (1997). Endoscopic Diagnosis of Hookworm Disease of the Duodenum. Journal of Clinical Gastroenterology, 24(2), 100-102.

8. Wahnschaffe, U., Ignatius, R., Loddenkemper, C., Liesenfeld, O.,
Muehlen, M., Jelinek, T., Schneider, T. (2007). Diagnostic value of endoscopy for the diagnosis of giardiasis and other intestinal diseases in patients with persistent diarrhea from tropical or subtropical areas. Scandinavian journal of gastroenterology, 42(3), 391-396.

9. Bhaijee, F., Subramony, C., Tang, S.J., \& Pepper, D. J. (2011). Human immunodeficiency virus-associated gastrointestinal disease: common endoscopic biopsy diagnoses. Pathology research international, 2011.

10.Garcia, L. S. (2002). Laboratory identification of the microsporidia. $J$ Clin Microbiol, 40(6), 1892-1901.

11.El-Badry, A. A., Al-Ali, K. H., \& Mahrous, A.-R. S. (2010). Molecular Identification \& Prevalence Of Giardia Lamblia \& Cryptosporidium In Duodenal Aspirate In Al-Madinah. microscopy, 14, 15.

12.Blakelock, R. T., \& Beasley, S. W. (2003). Infection and the gut. Seminars in Pediatric Surgery, 12(4), 265-274.

13.Mahdi, N., \& Taha, S. (2002). The Efficiency of Duodenal Aspirate in the Diagnosis of Parasitosis and Candidiasis. Qatar Medical Journal, 2002(2), 14.

14.Youssef, A. I., \& Uga, S. (2014). Review of parasitic zoonoses in Egypt. Tropical medicine and health, 42(1), 314.

15.Petersen, C. (1993). Cellular biology of Cryptosporidium parvum. Parasitology Today, 9(3), 87-91.

16.Fouad, S. A., Esmat, S., Basyoni, M. M. A., Farhan, M. S., \& Kobaisi, M. H. (2014). Molecular Identification of Giardia intestinalis in Patients with Dyspepsia. Digestion, 90(1), 63-71.

17.Jahani, M., Shafiee, R., Safavi, P., Rezaian, M., Amini, M., Ebrahimi, D. N., Shirzad, H. (2008). Prevalence of small bowel protozoan among dyspeptic patients who underwent upper gastrointestinal endoscopy (Tehran 2004-2006). Journal of Babol University of Medical Sciences, 10(1), 60-66.

18.Hill, D. R., \& Nash, T. E. (2007). Giardia lamblia. Principles and 
practice of infectious diseases, 2, 28882892.

19.Kaya, S., Cetin, E. S., Aridogan, B., Arikan, S., \& Demirci, M. (2007). Pathogenicity of Blastocystis hominis, a clinical reevaluation. Turkiye Parazitol Derg, 31(3), 184-187.

20.Sio, S. W. S., Puthia, M. K., Lee, A. S. Y., Lu, J., \& Tan, K. S. W. (2006). Protease activity of Blastocystis hominis. Parasitology Research, 99(2), 126-130.

21.Sulaiman, I. M., Ortega, Y., Simpson, S., \& Kerdahi, K. (2014). Genetic characterization of humanpathogenic Cyclospora cayetanensis parasites from three endemic regions at the $18 \mathrm{~S}$ ribosomal RNA locus. Infection, Genetics and Evolution, 22, 229-234.

22.Zimmer, S. M., Schuetz, A. N., \& Franco-Paredes, C. (2007). Efficacy of Nitazoxanide for Cyclosporiasis in Patients with Sulfa Allergy. Clinical infectious diseases, 44(3), 466-467.

23.Connor, B. A., Shlim, D. R., Scholes, J. V., Rayburn, J. L., Reidy, J., \& Rajah, R. (1993). Pathologic changes in the small bowel in nine patients with diarrhea associated with a coccidia-like body. Annals of Internal Medicine, 119(5), 377-382. 